\title{
Dynamics of microemulsions bridged with hydrophobically end-capped star polymers studied by neutron spin-echo
}

Cite as: J. Chem. Phys. 140, 034902 (2014); https://doi.org/10.1063/1.4861894

Submitted: 22 October 2013 . Accepted: 30 December 2013 . Published Online: 21 January 2014

I. Hoffmann, Paula Malo de Molina, B. Farago, P. Falus, Christoph Herfurth, André Laschewsky, and M. Gradzielski

\section{ARTICLES YOU MAY BE INTERESTED IN}

Structure and dynamics of polyelectrolyte surfactant mixtures under conditions of surfactant excess

The Journal of Chemical Physics 145, 124901 (2016); https://doi.org/10.1063/1.4962581

Dynamics of small unilamellar vesicles

The Journal of Chemical Physics 148, 104901 (2018); https://doi.org/10.1063/1.5009424

Neutron scattering studies on short- and long-range layer structures and related dynamics in imidazolium-based ionic liquids

The Journal of Chemical Physics 149, 054502 (2018); https://doi.org/10.1063/1.5037217

\section{Lock-in Amplifiers up to $600 \mathrm{MHz}$}
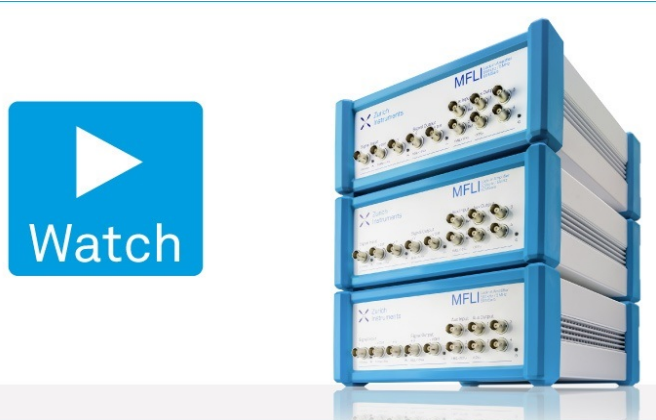

J. Chem. Phys. 140, 034902 (2014); https://doi.org/10.1063/1.4861894

140,034902

(C) 2014 AIP Publishing LLC. 


\title{
Dynamics of microemulsions bridged with hydrophobically end-capped star polymers studied by neutron spin-echo
}

\author{
I. Hoffmann, ${ }^{1,2, a)}$ Paula Malo de Molina, ${ }^{1, b)}$ B. Farago, ${ }^{2}$ P. Falus, ${ }^{2}$ Christoph Herfurth, ${ }^{3}$ \\ André Laschewsky, ${ }^{3}$ and M. Gradzielski ${ }^{1, c)}$ \\ ${ }^{1}$ Stranski-Laboratorium für Physikalische und Theoretische Chemie, Institut für Chemie, \\ Technische Universität Berlin, Straße des 17. Juni 124, Sekr. TC 7, D-10623 Berlin, Germany \\ ${ }_{2}^{2}$ Institut Max von Laue-Paul Langevin (ILL), F-38042 Grenoble Cedex 9, France \\ ${ }^{3}$ Fraunhofer Institut für Angewandte Polymerforschung IAP, Geiselbergstraße 69, 14476 Potsdam-Golm, \\ Germany
}

(Received 22 October 2013; accepted 30 December 2013; published online 21 January 2014)

\begin{abstract}
The mesoscopic dynamical properties of oil-in-water microemulsions (MEs) bridged with telechelic polymers of different number of arms and with different lengths of hydrophobic stickers were studied with neutron spin-echo (NSE) probing the dynamics in the size range of individual ME droplets. These results then were compared to those of dynamicic light scattering (DLS) which allow to investigate the dynamics on a much larger length scale. Studies were performed as a function of the polymer concentration, number of polymer arms, and length of the hydrophobic end-group. In general it is observed that the polymer bridging has a rather small influence on the local dynamics, despite the fact that the polymer addition leads to an increase of viscosity by several orders of magnitude. In contrast to results from rheology and DLS, where the dynamics on much larger length and time scales are observed, NSE shows that the linear polymer is more efficient in arresting the motion of individual ME droplets. This finding can be explained by a simple simulation, merely by the fact that the interconnection of droplets becomes more efficient with a decreasing number of arms. This means that the dynamics observed on the short and on the longer length scale depend in an opposite way on the number of arms and hydrophobic stickers. (ㅇ 2014 AIP Publishing LLC. [http://dx.doi.org/10.1063/1.4861894]
\end{abstract}

\section{INTRODUCTION}

Microemulsions (MEs) are isotropic, thermodynamically stable mixtures of oil and water stabilized with surfactant and potentially a cosurfactant. Depending on their composition, different structures are found: Oil-in-water $(\mathrm{O} / \mathrm{W})$ droplets, water-in-oil (W/O) droplets, or bicontinuous sponge phases. ${ }^{1-5}$ The ability to disperse hydrophobic substances in an aqueous solution in a thermodynamically stable fashion gives them a wide range of applications but the generally low viscosity of (W/O) MEs limits their application to a certain extent. To increase and control the viscosity, the addition of a thickening agent is needed. Quite commonly conventional polymeric thickeners are used for that purpose, which increase in general the viscosity of the solvent. ${ }^{6} \mathrm{Al}-$ ternatively, it is possible to control the rheological properties by cross linking the ME droplets with bridging amphiphilic polymers. ${ }^{7-14}$ If droplet concentration and polymer length are chosen appropriately, the viscosity can be increased dramatically by the addition of relatively small amounts of these polymers, i.e., increases by 3-4 orders of magnitude can easily be achieved. ${ }^{7,9}$ The majority of these investigations was concerned with studying $\mathrm{O} / \mathrm{W}$ microemulsions but in the case of

\footnotetext{
a) ingo.hoffmann@tu-berlin.de

b) Present address: Department of Chemical Engineering, University of California Santa Barbara, 3357 Engineering II, Santa Barbara, California 93106, USA

c) michael.gradzielski@tu-berlin.de
}

hydrophobic polymers with a hydrophilic end-cap, bridging aqueous droplets in W/O MEs has also been investigated. ${ }^{15}$ A potential problem with all these approaches is that the polymer may decrease the ME's stability or may otherwise change its properties in an unfavorable fashion, as, for instance, seen for the case of adding polymers to $\mathrm{W} / \mathrm{O} \mathrm{mi}-$ croemulsion droplets. ${ }^{15}$ In particular, it should be noted that basically all of these studies have been limited to the case of bifunctional, linear polymers.

In a recent study, ${ }^{16}$ the ability of hydrophobically endcapped poly(N,N-dimethylacrylamide) (PDMA) polymers with different numbers of arms to bridge a tetradecyl dimethyl amine oxide (TDMAO)/decane ME was investigated using small angle neutron scattering (SANS), rheology, and dynamic light scattering (DLS). These polymers can be efficiently synthesized by reversible addition-fragmentation chain transfer (RAFT) polymerization. ${ }^{17}$ It was found that they are able to form networks with the ME droplets and that the viscosity strongly increases with the length of the hydrophobic end-cap, leading to viscosity increases of up to six orders of magnitude. An important finding here was that the structure of the droplets was retained even for the addition of rather large amounts of hydrophobically modified polymer. The main finding was that the polymer introduced an increasing repulsive interaction between the microemulsion droplets with increasing polymer concentration. ${ }^{16}$

In contrast to this rather simple structural behavior, the dynamics of these polymer/microemulsion mixtures is rather 
complex. The autocorrelation functions measured with DLS showed a complex pattern with 3 modes. The fastest mode was the translational diffusion of the ME droplets, while 2 additional slow modes relate to the lifetime of the junctions between droplets and polymers and the relaxation of clusters formed by bridging many ME droplets. It was also found that polymers with a larger number of arms are more efficient in bridging the droplets, as indicated by a stronger increase in viscosity and pronouncedly larger amplitudes for the cluster relaxation modes observed in DLS.

However, DLS can only monitor the dynamic response on rather large length scales on the size of super structures in the mixture, but not on the natural length scale of the microemulsion. However, this is important information for understanding the dynamics on a mesoscopic level, as it might, for instance, be relevant for understanding the properties of such polymer modified microemulsions with respect to the release and transport of solubilized molecules. The method of choice for obtaining such mesoscopic information is neutron spin-echo (NSE) that observes dynamics on a more local scale, i.e., in the range of 2-40 nm. Accordingly in this work, we employed NSE to gain a much better understanding of the impact of the bridging on the dynamics of the individual droplets, for which we chose the TDMAO/decane microemulsion, which was shown before to form well-defined and rather monodisperse microemulsion droplets. ${ }^{18}$ In that context we focussed on the effect of the number of bridging arms, the length of the hydrophobic sticker, and the number of hydrophobic stickers per microemulsion droplet. Understanding the dynamics as a function of the molecular architecture then would allow to gain further fundamental insight into the dynamics of such systems and to optimize formulations for a certain dynamic response of the bridging polymer and thereby tailor the properties of the mixed microemulsion/polymer systems.

\section{THEORETICAL BACKGROUND}

On the time scale of the NSE experiment membrane fluctuations of MEs can be observed, in particular around the minimum of the form factor, ${ }^{19-23}$ and NSE has not only been used to study O/W but also W/O and bicontinuous MEs. ${ }^{24-28}$ These fluctuations are governed by the bending elasticity of the system. The bending energy can be expressed as a Helfrich bending Hamiltonian ${ }^{29}$

$$
E=\int\left\{\left(c_{1}+c_{2}-2 c_{0}\right)^{2} \kappa / 2+\bar{\kappa} c_{1} c_{2}\right\} \mathrm{d} A+{ }^{1} N k_{B} T \Phi(\phi),
$$

where $\kappa$ is the mean bending modulus, $\bar{\kappa}$ the Gaussian modulus, $c_{0}$ the spontaneous curvature, $c_{1}$ and $c_{2}$ are the principal curvatures, and $A$ the total surface of the system. The second term accounts for the entropy and consists of the particle number density ${ }^{1} N$, Boltzmann's constant $k_{b}$, temperature $T$, and $\Phi(\phi)$ which was approximated by

$$
\Phi(\phi)=\frac{1}{\phi}(\phi \ln (\phi)+(1-\phi) \ln (1-\phi)),
$$

with the volume fraction $\phi$.
Starting from a sphere and expanding in terms of spherical harmonics to second order leads to a form factor for fluctuating spheres given by $\mathrm{y}^{21,30,31}$

$$
\begin{aligned}
P(q, R)= & P_{\text {stat }}(q, R)+P_{\text {stat }, \text { corr }}(q, R)+P_{\text {dyn }}(q, R) \\
P_{\text {stat }}(q, R)= & \left(\frac{4 \pi R^{2}}{q} j_{1}(q R)\right)^{2}, \\
P_{\text {stat }, \text { corr }}(q, R)= & \frac{(4 \pi)^{2} R^{2} j_{1}(q R)}{q}\left(2 R j_{0}(q R)-q R^{2} j_{1}(q R)\right) \\
& \times \sum_{l \geq 2} \frac{2 l+1}{4 \pi}\left\langle a_{l}^{2}\right\rangle, \\
P_{\text {dyn }}(q, R, t)= & (4 \pi)^{2} R^{4} \sum_{l \geq 2} \frac{2 l+1}{4 \pi} j_{l}^{2}(q R)\left\langle a_{l}(0) a_{l}(t)\right\rangle,
\end{aligned}
$$

where $R$ is the radius of the sphere, $q=4 \pi / \lambda \sin (\theta / 2)$ is the magnitude of the scattering vector with scattering angle $\theta$ and wavelength $\lambda$. The $j_{l}$ are the $l$ th order spherical Bessel functions. The second line is the form factor of a simple sphere and the third and fourth line are static and dynamic corrections accounting for size and shape fluctuations. The $l=0$ terms correspond to size fluctuations which are too slow to be seen in a NSE experiment. The $l=1$ terms correspond to diffusion which is taken into account separately. Therefore, the first (and for purposes of fitting in this paper last) terms in the sums are the $1=2$ terms. The correlator $\left\langle a_{l}(0) a_{l}(t)\right\rangle$ in the last line reduces to $\left\langle a_{l}^{2}\right\rangle$ in a static experiment. In a dynamic experiment it reads $\left\langle a_{l}^{2}\right\rangle \exp \left(-\Gamma_{l} t\right)$. The amplitudes of the fluctuations $\left\langle a_{l}^{2}\right\rangle$ are given by

$$
\begin{aligned}
\left\langle a_{l}^{2}\right\rangle= & R^{2} \\
& \times \frac{k_{B} T}{\kappa(l+2)(l-1)\left(l(l+1)-6+4 w-\frac{3 \bar{\kappa}}{\kappa}-\frac{3 k_{B} T}{4 \pi \kappa} \Phi(\phi)\right)},
\end{aligned}
$$

where $w=c_{0} R$. For the maximum droplet size, which is reached at emulsification failure, $w$ can be replaced by $w_{\max }=1+\bar{\kappa} /(2 \kappa)+k_{B} T /(8 \pi \kappa) \Phi(\phi)$.

The overall static scattering intensity that also accounts for fluctuations of the particle shape reads

$$
I(q)={ }^{1} N \times \Delta S L D^{2} \int_{0}^{\infty} f_{q}(q) \int_{0}^{\infty} f_{R}(R) P(q, R) \mathrm{d} R \mathrm{~d} q,
$$

with scattering length density $\triangle S L D$, size distribution $f_{R}(R)$, scattering vector distribution $f_{q}(q)$, and particle number density ${ }^{1} N$, which is related to the volume fraction by

$$
{ }^{1} N=\frac{\phi}{\int_{0}^{\infty} f_{R}(R)(4 \pi / 3) R^{3} \mathrm{~d} R} .
$$

For an equilibrium system, the polydispersity $p$ is related to the bending moduli by

$$
p^{2}=\frac{\sigma^{2}}{R^{2}}=\frac{k_{B} T}{8 \pi(2 \kappa+\bar{\kappa})+2 k_{B} T \Phi(\phi)},
$$

where $\sigma$ is the standard deviation. 
For both $f_{R}(R)$ and $f_{q}(q)$ a Gaussian distribution was chosen. The width of the former is given by Eq. (7). For all but the lowest $q$-valuesthe $q$ vector distribution on the IN15 NSE instrument is dominated by the wavelength distribution $\Delta \lambda / \lambda$ $=0.15$ FWHM.

The time scale of $\left\langle a_{0}(0) a_{0}(t)\right\rangle$, which would require exchange of individual surfactant molecules, is too slow to be seen in aNSE experiment and the dominant dynamic contribution aside from diffusion, and comes from the $l=2$ term in $P_{d y n}$ (Eq. (3)). Its relaxation rate is given by

$$
\Gamma_{2}=\frac{\kappa}{\eta R^{3}} \frac{4 w-3 \frac{\bar{\kappa}}{\kappa}-\frac{3 k_{B} T}{4 \pi \kappa} \Phi(\phi)}{Z(2)},
$$

where $\eta$ is the viscosity of the solvent and

$$
Z(l)=\frac{(2 l+1)\left(2 l^{2}+2 l-1\right)}{l(l+1)(l+2)(l-1)} .
$$

To correct for finite concentration effects, the translational diffusion coefficient is corrected by the hard sphere structure factor in the Percus-Yevick approximation $S_{H S}(q, R, \phi),{ }^{32,33}$

$$
D(q)=\frac{k_{B} T}{6 \pi \eta R S_{H S}(q, R, \phi)},
$$

so that the (unnormalized) intermediate scattering function for the ME reads

$$
\begin{aligned}
s(q, t)_{M E}= & \int f_{q}(q) \int f_{R}(R)\left(P_{\text {stat }}(q, R)\right. \\
& \left.+P_{\text {stat }, \text { corr }}(q, R)+P_{d y n}(q, R, t)\right) \exp \left(-D(q) q^{2} t\right) \\
& \times S_{H S}(q, R, \phi) \mathrm{d} R \mathrm{~d} q
\end{aligned}
$$

and the structure factor is taken into account in the local monodisperse approach, ${ }^{34}$ which has been successfully used in the description of scattering data from MEs before. ${ }^{35,36}$ It might be noted that in Eq. (10) the hydrodynamic factor $H(q)$ has been omitted, which, however, for such a rather dilute system can be neglected, as it has been observed before that for similar microemulsions $S(0)$ is a much larger correction than $H(0)^{37}$ and $H(q)$ for not too dense colloids has been found to be close to $1 .{ }^{38}$ If the ME droplets are bridged by the polymers large clusters are formed from bridged droplets. Their translational diffusion will be hindered and Eq. (11) would change to

$$
\begin{aligned}
s(q, t)_{C}= & \int f_{q}(q) \int f_{R}(R)\left(P_{\text {stat }}(q, R)\right. \\
& \left.+P_{\text {stat }, \text { corr }}(q, R)+P_{d y n}(q, R, t)\right) \exp \left(-D_{c}(q) q^{2} t\right) \\
& \times S_{H S}(q, R, \phi) \mathrm{d} R \mathrm{~d} q,
\end{aligned}
$$

where $D_{c}$ is the diffusion constant of the clusters, which is too slow (according to DLS ${ }^{16}$ on the order of $0.01 \AA^{2} / n s$ and below) to be detected on the NSE time scale, therefore $D_{c}$ $\approx 0$. However, over short distances (and times) the droplets in the cluster do not necessarily notice the constraints in their freedom of movement, which is taken into account by a factor $x_{c}$, and only over longer times they move together with the whole cluster. Also a certain fraction of the droplets may be free and not involved in any cluster at all, which is taken into account by $x_{\text {free }}$. The whole intermediate scattering function then reads

$$
\begin{aligned}
s(q, t)= & x_{\text {free }} s(q, t)_{M E}+\left(1-x_{\text {free }}\right) \\
& \times\left[s(q, t)_{c}\left(1-x_{c}\right)+x_{c} s(q, t)_{M E}\right] .
\end{aligned}
$$

Assuming the dynamics of the droplets remains unchanged otherwise, there is no way to differentiate between the fraction of droplets not involved in the clusters $x_{\text {free }}$ and the amplitude of droplet movement in the clusters $x_{c}$, so that both are summarized in the "dynamic fraction" $x_{d y n}=x_{\text {free }}+x_{c}(1$ $\left.-x_{\text {free }}\right)$ and the resulting intermediate scattering function reads

$$
s(q, t)=x_{d y n} s(q, t)_{M E}+\left(1-x_{d y n}\right) s(q, t)_{C} .
$$

Aside from the contribution due to membrane fluctuations which are still present in the droplets in the clusters, this corresponds to the intermediate scattering function of the ME with an added constant background $\left(1-x_{d y n}\right)$ and dividing $s(q, t)$ by $s(q, 0)$ yields the normalized intermediate scattering function $S(q, t)$.

\section{MATERIALS AND METHODS}

\section{A. Materials}

Tetradecyl dimethyl amine oxide (TDMAO, $\mathrm{C}_{14} \mathrm{H}_{29} \mathrm{~N}\left(\mathrm{CH}_{3}\right)_{2} \mathrm{O}$ ) was a gift from Huntsman (Empigen $\mathrm{OH} 25,24 \%-26 \%)$. The solutions were freeze-dried and at the end still contained 2.5 wt. \% of water, as determined by Karl Fischer titration. The water content was accounted for in the preparation of TDMAO solutions. N-decane was obtained from Sigma Aldrich and used as received. $\mathrm{D}_{2} \mathrm{O}$ (99.9\% isotopic purity, Euriso-top, France) was used as a solvent for all samples. The samples were prepared by taking a solution containing $200 \mathrm{mM}$ of TDMAO. The appropriate amounts of oil and $\mathrm{D}_{2} \mathrm{O}$ were added to obtain the final composition of the ME with $100 \mathrm{mM}$ TDMAO/35 mM decane $/ \mathrm{D}_{2} \mathrm{O}$. The polymers were synthesized using reversible addition-fragmentation chain transfer polymerization. Details concerning synthesis and characterization of the polymers are given elsewhere. ${ }^{17}$ The polymers are based on hydrophilic poly(N,N-dimethylacrylamide) blocks and have 2, 3, or 4 arms, with an aliphatic end chain of 12 or 18 carbon units, respectively. See Table S I of the supplementary material ${ }^{39}$ for details.

The polymer containing MEs were prepared by mixing the appropriate amounts of MEs with varying amounts of polymer and mixing with a vortex mixer while heating $\left(\sim 60^{\circ} \mathrm{C}\right)$ to ensure complete homogenization. The number of stickers per droplet, $r$, is defined as the ratio between the number density of stickers, and the number density of droplets, making the assumption that all end stickers are located within the ME droplets. All polymer containing MEs had an excess of polymer to yield $r$ values of 2 or 4 and yielded highly viscous gels. Details concerning the structure and rheology of the samples can be found elsewhere. ${ }^{16}$ See Table S II of the supplementary material ${ }^{39}$ for the exact compositions. 


\section{B. Methods}

\section{Neutron spin-echo}

The NSE experiments were performed on the instrument IN15 of the ILL, Grenoble (France) with wavelengths of (longest Fourier times in parentheses) 22.8 (598 ns), 16 ( $207 \mathrm{~ns}$ ), and $10.5 \AA$ ( $52.6 \mathrm{~ns})$ to cover a $q$-range from 0.018 to $0.161 / \AA$. Details of the experiment and the method are explained elsewhere. ${ }^{40,41}$

\section{RESULTS AND DISCUSSION}

\section{A. Results}

As a starting point for the data analysis the initial slope of the intermediate scattering functions of the ME has been fitted with a single exponential to obtain an apparent diffusion coefficient $D_{\text {eff: }}$ :

$$
D_{e f f}=\frac{\mathrm{d} S(q, t)}{\mathrm{d} t} \frac{1}{q^{2}},
$$

or for any practical purposes $D_{\text {eff }}=\Delta S(q, t) / \Delta t \times 1 / q^{2}$ with a finite $\Delta t$. It is observed that $D_{\text {eff }}$ (see Fig. 1) shows a maximum at $0.131 / \AA$. This corresponds to $q R=\tan (q R)$ thus comes form the shape fluctuations of the droplets. ${ }^{22}$ The increase at low $q$ is due to the structure factor and the behavior can be well described by theory if we assume, that the hydrodynamic radius, determining translational diffusion is increased by a solvent shell. The solid line in Fig. 1 was calculated for the parameters that fitted best the whole NSE curves, which explains why they do not fit so perfectly to this set of data derived from the initial slope. The obtained radius of $32.2 \AA$ is in very good agreement with former investigation of the same system ${ }^{18,37}$ and the somewhat lower values reported in Ref. 16 can be attributed to the fact that in the latter work the experimental smearing was not accounted for explicitly which leads to an incorporation of these effects into the polydispersity, which in turn leads to a lowering of the apparent average radius. Finally the shell of $5.8 \AA$ can be explained

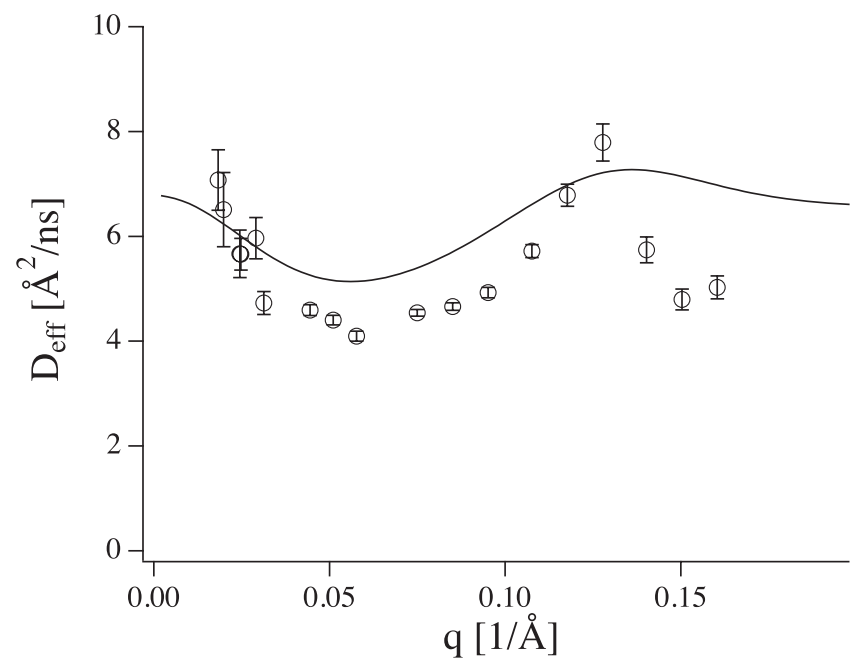

FIG. 1. Apparent diffusion coefficient $D_{\text {eff }}$ of TDMAO ME. The line is the effective diffusion coefficient resulting from Eqs. (11) and (15), with $\Delta t$ $=3 \mathrm{~ns}, R=32.2 \AA$, solvent shell $5.8 \AA, \kappa=1.5 k_{B} T, \bar{\kappa}=-2.4 k_{B} T$.

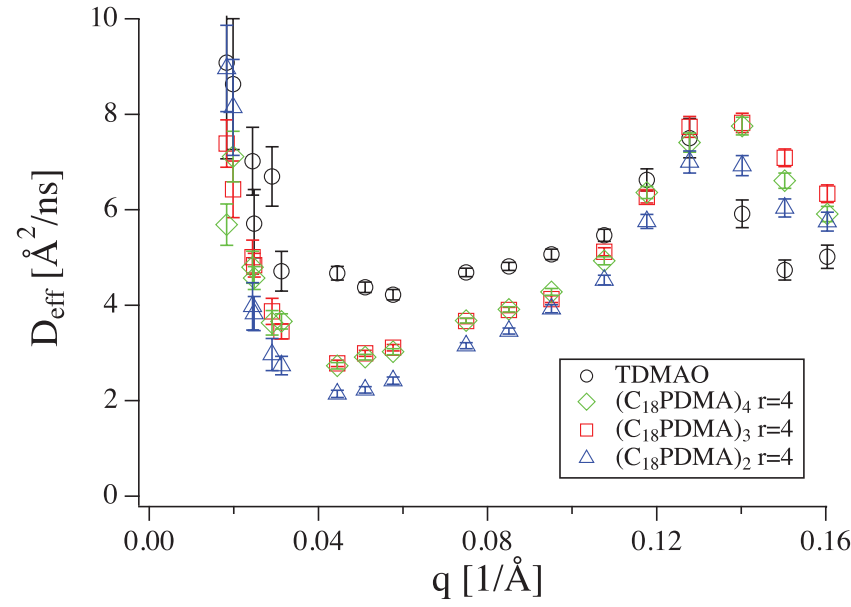

FIG. 2. Apparent diffusion coefficient of the samples indicated in the graph. The effect of polymer addition is mainly seen around $0.041 / \AA$ where $D_{\text {eff }}$ is lowered due to the presence of the polymer.

by a hydration shell of strongly bound water at the surface of the microemulsion droplets. A part of the remaining discrepancy can be attributed to the hydrodynamic factor $H(q)$, which has been omitted in the calculation of the theoretical curve in Fig. 1 as it should not have a great influence at the given concentration but would still decrease $D_{\text {eff }}$.

Adding the polymer to the ME it is seen that the high $q$ part $(q>0.11 / \AA)$ remains more or less unchanged upon addition of the polymer (see Fig. 2 and Fig. S1 of the supplementary material ${ }^{39}$ ). This means that the polymer does not affect the membrane rigidity of the microemulsion droplet. This is to be expected as the ratio of hydrophobic stickers per surfactant molecule is very low-even for $r=4$ just being about $2.5 \%$, so the amphiphilic interface remains effectively the same. At the three highest $q$ values the scattered intensity from the polymer itself starts to contribute which might explain the small difference.

At lower $q(q<0.11 / \AA) D_{\text {eff }}$ is significantly reduced by the addition of the polymer and the appearance of a second, significantly slower mode can be observed in the intermediate scattering function (see Fig. 3 and Fig. S2 of the supplementary material ${ }^{39}$ ). This is in qualitative agreement with previous findings from DLS. ${ }^{16}$ The slow mode is too slow for the NSE timescale and therefore it was taken into account as a constant term as described in Eq. (14). It might be noted that in all our analyses we employed a constant solvent viscosity of $1.132 \mathrm{mPas}$ thereby neglecting potential changes of it due to the presence of the water-soluble PDMA chains. This is not an easy to prove assumption but can be considered to be reasonable as for similar concentrations of PDMA one finds different values of $D(q)$ (Fig. S1 of the supplementary material ${ }^{39}$ ).

For a quantitative description, data from the pure ME were fitted with Eq. (11) using the same parameters for all $q$-values (see Fig. S3 of the supplementary material ${ }^{39}$ ). As it has been seen before that the ME droplets themselves remain unchanged upon addition of the polymer, ${ }^{16}$ the same parameters $(R, \kappa, \bar{\kappa})$ are used for all samples with added polymer and the only free parameter is $x_{d y n}$ (see Eq. (14)) and a good quantitative description is obtained for all samples (see Fig. S4 of 


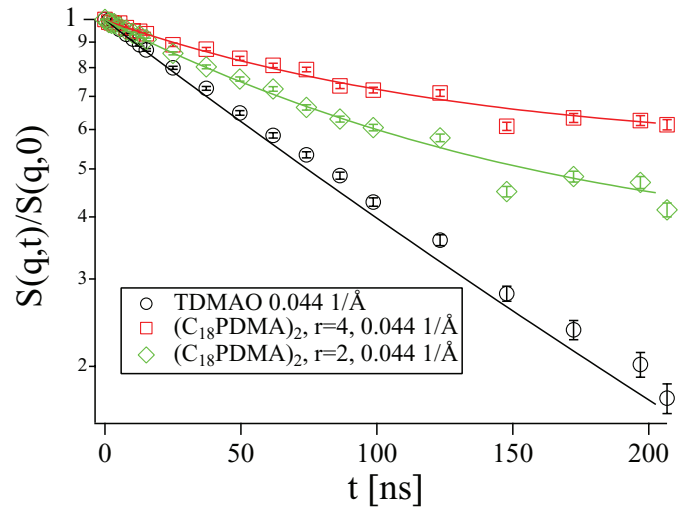

FIG. 3. $S(q, t)$ at $q=0.0441 / \AA$ for pure ME (black) and ME with added polymer ( $\left.\mathrm{C}_{18} \mathrm{PDMA}\right)_{2}, r=4$ (red squares) and $r=2$ (green diamonds), the relaxation is slowed down due to the polymer and the intermediate scattering function deviates from a simple exponential. Fits are according to Eq. (14) (added polymer) or Eq. (11) (pure ME).

the supplementary material ${ }^{39}$ for an example) and trends for the change of $x_{d y n}$ with polymer concentration, sticker length and the number of arms of the polymer can be deduced. The obtained data are displayed in Fig. 4 and one finds a value of $\sim 1$ for the largest $q$-values that decreases substantially for lower $q$. This can be explained such that when looking at short diffusion distances (large $q$ ) almost all droplets are freely diffusing. Only when the diffusion over longer distances (small $q$ ) is considered the restrictions by being in a cluster become felt by the microemulsion droplets thereby lowering the apparent value for $x_{d y n}$ (see also Fig. S2 of the supplementary material ${ }^{39}$ for this distance dependence).

Volino and Dianoux ${ }^{42}$ derived an expression for the intermediate scattering function of particles diffusing inside a sphere of a given diameter, which involves a $q$-dependent elastic contribution (which translates to a constant in the intermediate scattering function). Even though this is a rather crude approximation for the problem at hand, the high $q$ part of Fig. 4 can be described quite nicely for all samples, assuming the sphere has a diameter of $6.4 \mathrm{~nm}$ (see solid line in Fig. 4), which is roughly in agreement with the half end-to-

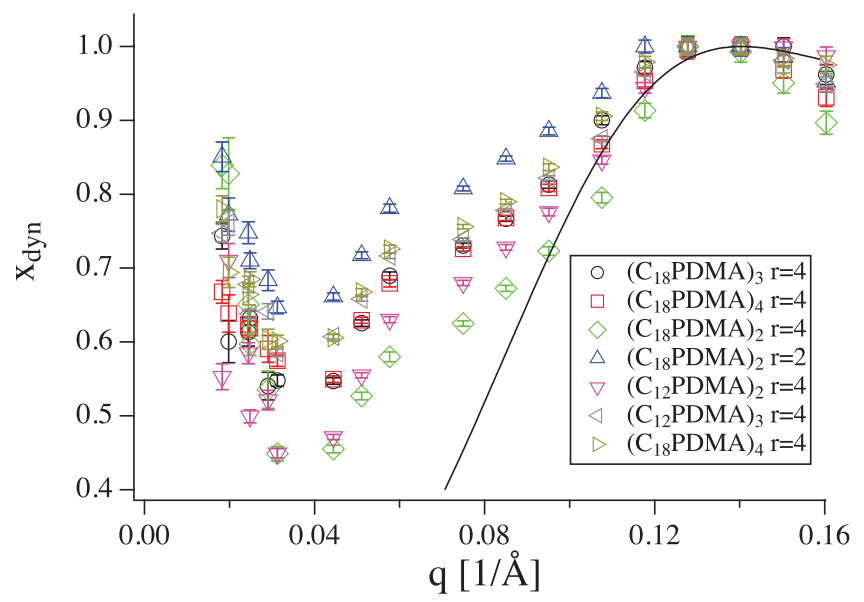

FIG. 4. $x_{d y n}$ as a function of $q$ for samples indicated in the graph. Black line: quasielastic contribution for diffusion in a sphere of $6.4 \mathrm{~nm}$ diameter according to Volino and Dianoux. ${ }^{42}$

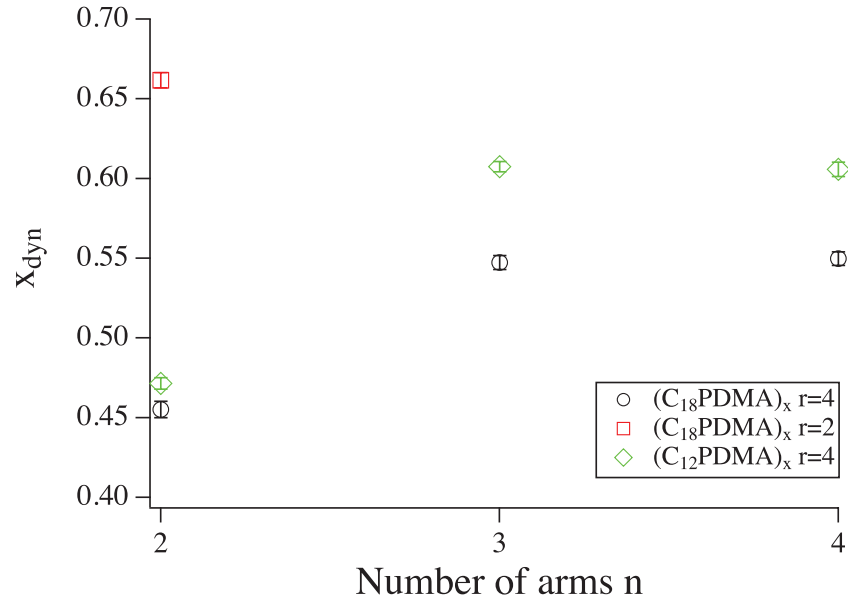

FIG. 5. $x_{d y n}$ as a function of the number of arms at $0.0441 / \AA$ for different polymers and polymer arm to droplet ratio $r$ (black circles: $\mathrm{C}_{18} \mathrm{PDMA}_{\mathrm{x}}, r$ $=4$; red squares: $\mathrm{C}_{18} \mathrm{PDMA}_{\mathrm{x}}, r=2$; green diamonds: $\mathrm{C}_{12} \mathrm{PDMA}_{\mathrm{x}}, r=4$ ); it can be seen that the 2-arm polymers are more efficient in arresting the diffusion of the ME. The $\mathrm{C}_{18}$-polymers are only slightly more efficient than the $\mathrm{C}_{12}$-polymers. For $\mathrm{C}_{18}, r=2$ only the 2 -arm polymer was measured.

end distance of 2 arms of the polymers (see Table S I of the supplementary material ${ }^{39}$ ), i.e., assuming a movement confined by the holding potential of the polymers with their stickers in the droplets. At lower $q$ the discrepancies between the actual soft potential and the hard wall used in the theory become obvious as the theoretical curve starts to deviate from the experimental values.

The obtained trends for $x_{d y n}$ with the number of arms, the sticker length, and the polymer concentration are summarized in Fig. 5. An increase of the polymer concentration significantly decreases $x_{d y n}$. Around $0.051 / \AA$ the constant background is roughly doubled when increasing the polymer concentration by a factor of 2 .

Comparing the values of $x_{d y n}$ for samples with different sticker length it can be seen that the sticker length has only a small influence on the dynamics of the ME droplets, which is in contrast to results from DLS ${ }^{16}$ where the amplitude of the slow modes greatly depends on the sticker length.

A very surprising result is the fact that the 2-arm polymer is more efficient in arresting the ME droplets which at first glance appears to be in disagreement with DLS, where both the slow mode relaxation times are longer with more arms and their amplitudes are higher, and with rheology where the viscosity is found to be higher for polymers with more arms.

\section{B. Simulations}

An attempt to explain at first glance contradictory results of DLS (higher amplitude of slow mode for more arms) and NSE (higher amplitude of slow mode with less arms) was done with the following simple approach: With more arms the clusters that are formed are actually larger due to their higher connectivity but in total, less droplets are involved in their formation, as there is a higher probability for the individual arms not to connect to a droplet or to connect to the same droplet as another arm of the same polymer as the number of arms is increased due to steric constraints. At low $q$ (DLS) the 
amplitude is related to the cluster size (number of droplets per cluster), whereas at high $q$ (NSE) the individual droplets are seen and hence the amplitude of the slow mode in NSE is related to the total number of droplets involved in any of the clusters.

This general behavior was simulated as follows: To a system of $d$ "droplets" to which an arbitrary amount of links can be established, $d \times r / n$ "polymers" with $n$ arms are added consecutively until a certain polymer arm to droplet ratio is reached.

Each polymer links to $n$ random droplets, where multiple arms of one polymer can link to the same droplet.

Based on the randomly determined droplets, either a new cluster is formed, new droplets are added to an existing cluster or existing clusters are united to form a new cluster. In the end the average cluster size and the number of droplets involved in clusters are calculated.

To account for the increased difficulty of the 2 nd, $3 \mathrm{rd}, \ldots, n$th arm to find a droplet, a probability $p_{\text {same }}\left(n_{i}\right)$ for the $n_{i}$ th arm to connect to the same droplet as the first arm was included, which in the framework of the model has the same effect as a dangling end. $p_{\text {same }}\left(n_{i}\right)$ increases linearly as $p_{\text {same }}\left(n_{i}\right)=\left(n_{i}-1\right) a$ (with a maximum value of 1$)$, which means that the first arm always finds a droplet and it becomes increasingly unlikely for the following arms and $a$ determines how fast the difficulty to find another droplet increases with the arm number. By doing so, the number of polymer arms introduced into the system is reduced from $N_{\text {arms }}=d \times r$ to an effective number of arms $N_{\text {arms,eff }}=d \times r \times(1$ $\left.-p_{\text {same }, a v}(n, a)\right)$, where $p_{\text {same, } a v}=n^{-1} \sum_{n_{i}=1}^{n}\left(n_{i}-1\right) a$ is the average value of $p_{\text {same }}$ for a given type of polymer. At the same time the effective number of arms per polymer is changed from $n$ to $n_{\text {eff }}=\sum_{n_{i}=1}^{n} 1-p_{\text {same }}\left(n_{i}\right)$. To give an example, with $d=1000, r=4$, and $a=0.1$, the resulting

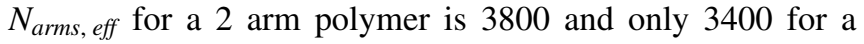
4 arm polymer, in other words the overall number of droplets involved in aggregates is lower for the 4 arm polymer. However, $n_{\text {eff }}$ for the 4 arm polymer is still higher (3.4 as opposed to 1.9 for the 2 arm polymer) and therefore the number of droplets per cluster is still higher. For comparison between different numbers of arms $p_{\text {same }, a v}^{\prime}=(n-1)^{-1} \sum_{n_{i}=2}^{n}$ $\left(n_{i}-1\right) a$ is used, which is the average probability for dangling ends or connections to the same droplets in all but the first arm. The model completely ignores geometry or the level of connectivity between the droplets and the steric constraints only comes into play via $p_{\text {same }}$.

For every value of $n$ and $a 25$ simulations with $r=4$ and $d=500000$ were performed and the results were averaged. The error bars are the standard deviations. Fig. S5 of the supplementary material ${ }^{39}$ shows the average cluster size for different values of $a$, while Fig. S6 of the supplementary material $^{39}$ shows the overall number of droplets involved in clusters. Figs. 6 and 7 show the average cluster size and number of droplets in clusters as a function of $p_{\text {same }}^{\prime}$.

The simulations show that indeed over a considerable range of different values of $a$ for a given value the average cluster size is bigger for a higher number of arms, whereas the overall number of droplets involved in the aggregates is lower for higher $n$. Furthermore the simulations also show

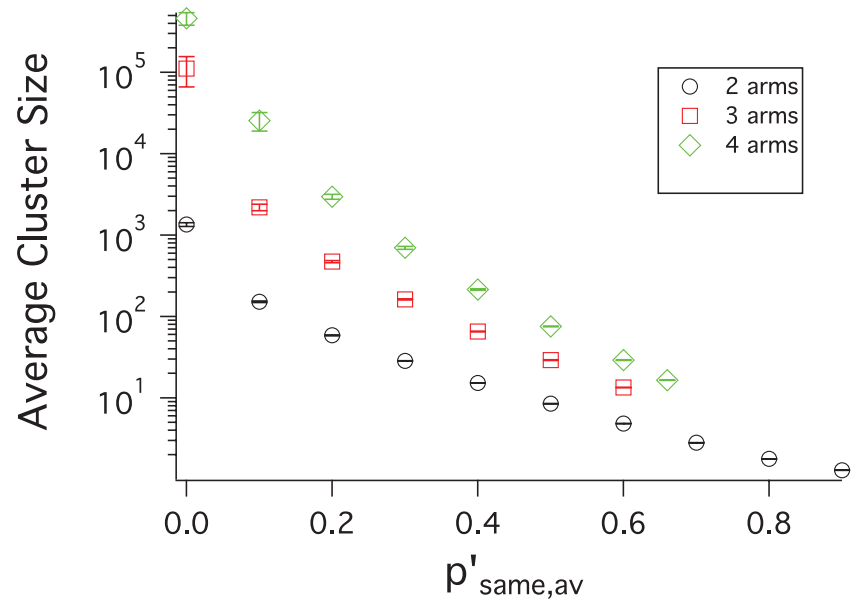

FIG. 6. Average cluster size for 2-, 3-, and 4-arm polymer for $r=4$ as a function of $p_{\text {same, } a v}^{\prime}$. The clusters are larger with a higher number of arms.

that the behavior of the 3 -arm polymer is closer to that of the 4-arm than the 2-arm polymer, which is in agreement with the experimental observations (Fig. 5). Thus, the simulations support our hypothesis that the difference in the amplitude of the slow mode observed in DLS and NSE is indeed due to the fact that NSE monitors individual droplets while DLS observes the clusters.

\section{Discussion}

We report the first neutron spin-echo measurements on microemulsions bridged with telechelic, hydrophobically end-caped polymers with different numbers of arms. NSE reveals important details on the length scale of the droplets, which are not to be observed with other methods.

It is found that the bridging has hardly any influence on the fluctuations of the amphiphilic monolayer of the $\mathrm{ME}$ droplets, i.e., their bending rigidity remains unaffected. This finding complements earlier SANS measurements, which showed that the structure of the MEs remains unchanged under these conditions. ${ }^{16}$

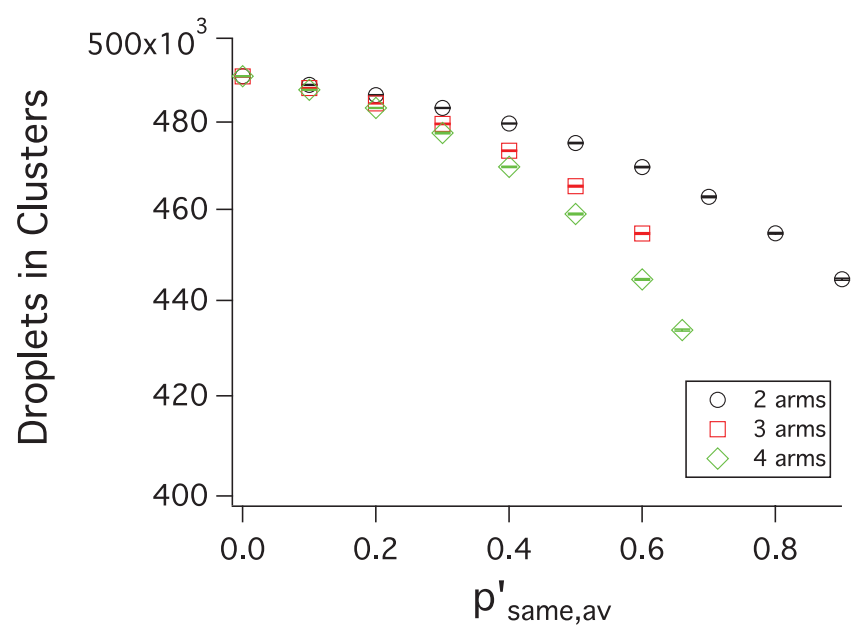

FIG. 7. Overall number of droplets in clusters for 2-, 3-, and 4-arm polymers for $r=4$ as a function of $p_{\text {same, } a v}^{\prime}$. The number of droplets in clusters drops faster with a higher number of arms. 

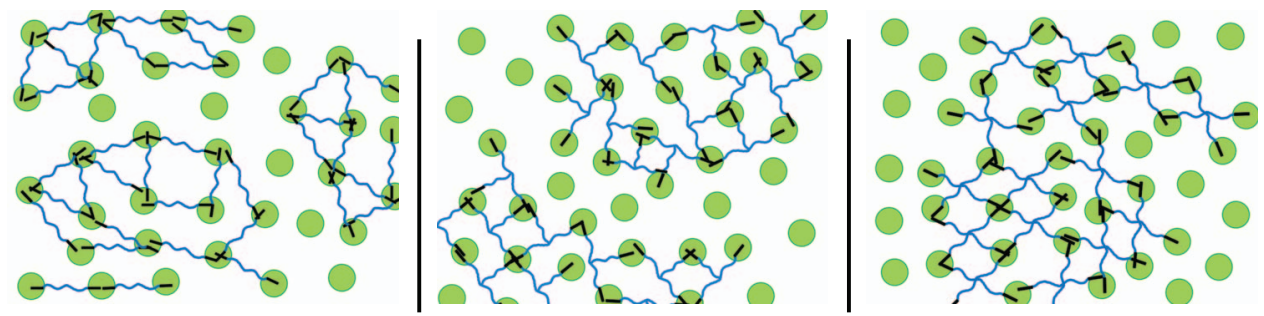

FIG. 8. Scheme of ME droplets linked with 2- (left), 3- (center), and 4-arm polymers (right). With a lower number of arms there are less free ME droplets and hence the number of droplets in clusters is lower (see Fig. 7) but the average cluster size is decreased i.e., an individual cluster consists of a lower number of ME droplets (see Fig. 6).

More surprising are the findings that the linear (2-arm) polymer is more efficiently arresting the dynamics of the ME droplets than 3- and 4-arm polymers, despite the fact that the macroscopic viscosity is much higher for polymers with more arms (at a given concentration of end groups). In addition, the length of the hydrophobic end-cap has only very little influence on the ME dynamics despite a strong increase in viscosity and structural relaxation time observed in rheology. The difference can be explained when considering the length scales which the different methods observe. Unlike DLS where mostly large clusters of many ME droplets are observed, and unlike rheology, where macroscopic properties are measured, NSE observes the dynamics of individual ME droplets. In that context it might also be noted that the residence time of a sticker in a hydrophobic domain is already for a $\mathrm{C}_{12}$ chain typically in the range of $10 \mu \mathrm{s}^{43}$ and therefore for all systems investigated here we can assume that on the NSE time-scale the stickers remain contained in the microemulsion droplets and their dynamics can be neglected.

On the level of individual droplets, the 2-arm polymer is more efficient in arresting the diffusion of the droplets as there are less conformational constraints imposed on the chain, which would keep it from finding a droplet, as confirmed by a simple simulation. The fact that the viscosity of 3 and 4-arm polymers containing systems is nonetheless much higher, is due to the individual junction on average linking more droplets in that case; thereby the probability for breaking the network is reduced, explaining the increased elastic properties. Fig. 8 illustrates this finding. This hypothesis is supported by a simplistic simulation (see also the supplementary material ${ }^{39}$ for further details), which shows that the average cluster size grows with the number of arms, even though the overall number of ME droplets involved in the clusters is reduced, as there is a higher probability for the arms not to find a different droplet than for the other arms of the same polymer (or no droplet at all). This qualitatively explains our results. The experiments and simulation show that the amplitude of the dynamics of the individual droplets (monitored by NSE) has an opposite dependence on the number of arms as that of their clusters (monitored by DLS). Therefore the NSE experiments allow to shed additional light on the dynamics of such complex colloidal systems. They allow to understand their dynamics, starting from that of the small individual microemulsion droplets to the large clusters comprising thousands of them. However, such fundamental and detailed knowledge of the dynamics is of general relevance in order to control and optimize the properties of such systems, for instance, for purposes of controlled release and delivery of active ingredients such as drugs.

\section{ACKNOWLEDGMENTS}

Financial support from the BMBF Project 05K10KT1 (I.H.) is gratefully acknowledged as well as allocation of beamtime by the ILL. This work was further supported by the DFG (Grant No. GR1030/9-1) and PMdM (Grant No. LA611/8-1). I.H. wishes to thank N. Goldin for fruitful discussions. We would also like to thank the two referees whose comments certainly helped to improve this manuscript further.

${ }^{1}$ J. H. Schulman, W. Stoeckenius, and L. M. Prince, "Mechanism of formation and structure of micro emulsions by electron microscopy," J. Phys. Chem. 63, 1677-1680 (1959).

${ }^{2}$ P. G. de Gennes and C. Taupin, "Microemulsions and the flexibility of oil/water interfaces," J. Phys. Chem. 86, 2294-2304 (1982).

${ }^{3}$ M. Kahlweit and R. Strey, "Phase behavior of ternary systems of the type h2o-oil-nonionic amphiphile (microemulsions)," Angew. Chem. Int. Ed. Engl. 24, 654-668 (1985).

${ }^{4}$ Microemulsions and Related Systems: Formulation, Solvency, and Physical Properties, Surfactant Science Series Vol. 30, edited by M. Bourrel and R. S. Schechter (Marcel Dekker, Inc., 1988).

${ }^{5}$ D. Langevin, "Micelles and microemulsions," Annu. Rev. Phys. Chem. 43, 341-369 (1992).

${ }^{6}$ R. Lapasin, M. Grassi, and N. Coceani, "Effects of polymer addition on the rheology of o/w microemulsions," Rheol. Acta 40, 185-192 (2001).

${ }^{7}$ M. Gradzielski, A. Rauscher, and H. Hoffmann, "Hydrophobically crosslinked micellar solutions: Microstructure and properties of the solutions," J. Phys. IV France 03, C1-65-C1-79 (1993).

${ }^{8} \mathrm{~W}$. Meier, "Structured polymer networks from o/w-microemulsions and liquid crystalline phases," Langmuir 12, 6341-6345 (1996).

${ }^{9}$ H. Bagger-Jörgensen, L. Coppola, K. Thuresson, U. Olsson, and K. Mortensen, "Phase behavior, microstructure, and dynamics in a nonionic microemulsion on addition of hydrophobically end-capped poly(ethylene oxide)," Langmuir 13, 4204-4218 (1997).

${ }^{10}$ M. Filali, R. Aznar, M. Svenson, G. Porte, and J. Appell, "Swollen micelles plus hydrophobically modified hydrosoluble polymers in aqueous solutions: Decoration versus bridging. A small angle neutron scattering study," J. Phys. Chem. B 103, 7293-7301 (1999).

${ }^{11}$ E. Michel, M. Filali, R. Aznar, G. Porte, and J. Appell, "Percolation in a model transient network: Rheology and dynamic light scattering," Langmuir 16, 8702-8711 (2000).

${ }^{12}$ F. E. Antunes, K. Thuresson, B. Lindman, and M. G. Miguel, "A rheological investigation of the association between a non-ionic microemulsion and hydrophobically modified PEG: Influence of polymer architecture," Colloids Surf., A 215, 87-100 (2003).

${ }^{13}$ S. Maccarrone, H. Frielinghaus, J. Allgaier, D. Richter, and P. Lindner, "SANS study of polymer-linked droplets," Langmuir 23, 9559-9562 (2007).

${ }^{14}$ P. I. Hurtado, L. Berthier, and W. Kob, "Heterogeneous diffusion in a reversible gel," Phys. Rev. Lett. 98, 135503 (2007).

${ }^{15}$ T. Blochowicz, C. Gägelein, T. Spehr, M. Müller, and B. Stühn, "Polymerinduced transient networks in water-in-oil microemulsions studied by 
small-angle x-ray and dynamic light scattering," Phys. Rev. E 76, 041505 (2007).

${ }^{16}$ P. Malo de Molina, C. Herfurth, A. Laschewsky, and M. Gradzielski, "Structure and dynamics of networks in mixtures of hydrophobically modified telechelic multiarm polymers and oil in water microemulsions," Langmuir 28, 15994-16006 (2012).

${ }^{17}$ C. Herfurth, P. Malo de Molina, C. Wieland, S. Rogers, M. Gradzielski, and A. Laschewsky, "One-step raft synthesis of well-defined amphiphilic star polymers and their self-assembly in aqueous solution," Polym. Chem. 3, 1606-1617 (2012).

${ }^{18}$ M. Gradzielski, H. Hoffmann, and D. Langevin, "Solubilization of decane into the ternary system tdmao/1-hexanol/water," J. Phys. Chem. 99, 1261212623 (1995).

${ }^{19}$ B. Farago, M. Monkenbusch, K. Goecking, D. Richter, and J. Huang, "Dynamics of microemulsions as seen by neutron spin echo," Phys. B 213-214, 712-717 (1995)

${ }^{20}$ M. Gradzielski, D. Langevin, and B. Farago, "Experimental investigation of the structure of nonionic microemulsions and their relation to the bending elasticity of the amphiphilic film," Phys. Rev. E 53, 3900-3919 (1996).

${ }^{21}$ B. Farago and M. Gradzielski, "The effect of the charge density of microemulsion droplets on the bending elasticity of their amphiphilic film," J. Chem. Phys. 114, 10105-10122 (2001).

${ }^{22}$ T. Hellweg, M. Gradzielski, B. Farago, and D. Langevin, "Shape fluctuations of microemulsion droplets: A neutron spin-echo study," Colloids Surf., A 183-185, 159-169 (2001).

${ }^{23}$ M. Nagao and H. Seto, "Concentration dependence of shape and structure fluctuations of droplet microemulsions investigated by neutron spin echo spectroscopy,” Phys. Rev. E 78, 011507 (2008).

${ }^{24}$ T. Spehr, B. Frick, I. Grillo, P. Falus, M. Müller, and B. Stühn, "Structure and dynamics of reverse micelles containing supercooled water investigated by neutron scattering," Phys. Rev. E 79, 031404 (2009).

${ }^{25}$ M. Monkenbusch, O. Holderer, H. Frielinghaus, D. Byelov, J. Allgaier, and D. Richter, "Bending moduli of microemulsions; comparison of results from small angle neutron scattering and neutron spin-echo spectroscopy," J. Phys.: Condens. Matter 17, S2903-2909 (2005).

${ }^{26}$ S. Wellert, H. Altmann, A. Richardt, A. Lapp, P. Falus, B. Farago, and T. Hellweg, "Dynamics of the interfacial film in bicontinuous microemulsions based on a partly ionic surfactant mixture: A neutron spin-echo study," Eur. Phys. J. E 33, 243-250 (2010).

${ }^{27}$ B. Farago, "Recent developments and applications of NSE in soft matter," Curr. Opin. Colloid Interface Sci. 14, 391-395 (2009).

${ }^{28}$ O. Holderer, M. Klostermann, M. Monkenbusch, R. Schweins, P. Lindner, R. Strey, D. Richter, and T. Sottmann, "Soft fluctuating surfactant membranes in supercritical $\mathrm{CO}_{2}$-microemulsions," Phys. Chem. Chem. Phys. 13, 3022-3025 (2011)
${ }^{29}$ W. Helfrich, "Elastic properties of lipid bilayers-theory and possible experiments," Z. Naturforsch, C: J. Biosci. C 28, 693-703 (1973).

${ }^{30}$ S. A. Safran, "Fluctuations of spherical microemulsions," J. Chem. Phys. 78, 2073-2076 (1983).

${ }^{31}$ S. T. Milner and S. A. Safran, "Dynamical fluctuations of droplet microemulsions and vesicles," Phys. Rev. A 36, 4371-4379 (1987).

${ }^{32}$ J. K. Percus and G. J. Yevick, "Analysis of classical statistical mechanics by means of collective coordinates," Phys. Rev. 110, 1-13 (1958).

${ }^{33}$ P. N. Pusey, "The dynamics of interacting brownian particles," J. Phys. A: Math. Gen. 8, 1433-1440 (1975).

${ }^{34}$ J. S. Pedersen, "Determination of size distribution from small-angle scattering data for systems with effective hard-sphere interactions," J. Appl. Crystallogr. 27, 595-608 (1994).

${ }^{35}$ T. Foster, T. Sottmann, R. Schweins, and R. Strey, "Small-angle neutron scattering from giant water-in-oil microemulsion droplets. I. ternary system," J. Chem. Phys. 128, 054502-13 (2008).

${ }^{36}$ T. Foster, T. Sottmann, R. Schweins, and R. Strey, "Small-angle-neutronscattering from giant water-in-oil microemulsion droplets. II. polymerdecorated droplets in a quaternary system," J. Chem. Phys. 128, 064902-18 (2008).

${ }^{37} \mathrm{M}$. Gradzielski and H. Hoffmann, "Influence of charges on structure and dynamics of an o/w microemulsion. Effect of admixing ionic surfactants," J. Phys. Chem. 98, 2613-2623 (1994).

${ }^{38}$ G. Grübel, D. L. Abernathy, D. O. Riese, W. L. Vos, and G. H. Wegdam, "Dynamics of dense, charge-stabilized suspensions of colloidal silica studied by correlation spectroscopy with coherent x-rays," J. Appl. Cryst. 33, 424-427 (2000)

${ }^{39}$ See supplementary material at http://dx.doi.org/10.1063/1.4861894 for more information on the polymers, the exact composition of samples and additional data.

${ }^{40}$ P. Schleger, B. Alefeld, J. Barthelemy, G. Ehlers, B. Farago, P. Giraud, C. Hayes, A. Kollmar, C. Lartigue, F. Mezei, and D. Richter, "The longwavelength neutron spin-echo spectrometer IN15 at the Institut LaueLangevin,” Phys. B 241-243, 164-165 (1997).

${ }^{41}$ F. Mezei "Neutron spin echo," in Lecture Notes in Physics, edited by Ferenc Mezei (Springer, Berlin/Heidelberg, 1980), Vol. 128, pp. 1-26.

${ }^{42}$ F. Volino and A. J. Dianoux, "Neutron incoherent scattering law for diffusion in a potential of spherical symmetry: general formalism and application to diffusion inside a sphere," Mol. Phys. 41, 271-279 (1980).

${ }^{43}$ E. A. G. Aniansson, S. N. Wall, M. Almgren, H. Hoffmann, I. Kielmann, W. Ulbricht, R. Zana, J. Lang, and C. Tondre, "Theory of the kinetics of micellar equilibria and quantitative interpretation of chemical relaxation studies of micellar solutions of ionic surfactants," J. Phys. Chem. 80, 905922 (1976). 